\title{
Effects of Oral Supplementation with Pyrroloquinoline Quinone on Stress, Fatigue, and Sleep
}

\author{
Masahiko Nakano ${ }^{*}$, Tetsuro Yamamoto ${ }^{2}$, Hisayoshi Okamura ${ }^{3}$, Akira Tsuda ${ }^{4}$, \\ and Yasuyuki Kowatari ${ }^{5}$
}

${ }^{1}$ Niigata Research Laboratory, Mitsubishi Gas Chemical Company, Inc., 182 Shinwari, Tayuhama, Kita-ku, Niigata 950-3112, Japan; ${ }^{2}$ TTC Co., Ltd., 1-20-2 Ebisunishi, Shibuya-ku, Tokyo 150-0021, Japan; ${ }^{3}$ Cognitive and Molecular Institute of Brain Diseases, Kurume University, 67 Asahi-machi, Kurume, Fukuoka 830-0011, Japan; ${ }^{4}$ Department of Psychology, Kurume University, 1635 Miimachi, Kurume 839-8502, Japan; ${ }^{5}$ Ueno Clinic, Aisei Hospital, 2-18-6 Higashiueno, Taito-ku, Tokyo, 110-0015, Japan

*Corresponding author: Masahiko Nakano, Niigata Research Laboratory, Mitsubishi Gas Chemical Company, Inc., 182 Shinwari, Tayuhama, Kita-ku, Niigata 950-3112, Japan

Submission date: July 20, 2012, Acceptance date: August 28, 2012; Publication date: August 31,2012

\begin{abstract}
:
Seventeen adult male and female subjects participated in a clinical trial using an open-label trial to evaluate the effectiveness of pyrroloquinoline quinone (PQQ) on stress, fatigue, quality of life and sleep. They ingested $20 \mathrm{mg}$ of PQQ daily for 8 weeks. Changes in stress, fatigue, quality of life measures and sleep were evaluated using various inventories and questionnaires. For example, the results of the Profile of Mood States-Short Form revealed that all six measures of vigor, fatigue, tension-anxiety, depression, anger-hostility and confusion were significantly improved following PQQ administration compared with scores for those measures before administration of PQQ. Measures for quality of life, appetite, sleep, obsession and pain, also improved significantly. The results of the Oguri-Shirakawa-Azumi Sleep Inventory (Middle Aged and Aged version) showed significant improvement in sleepiness at awakening, sleep onset and maintenance, and sleep duration. For validation, the Pittsburgh Sleep Quality Index Japanese version also showed significant improvement in sleep-related behavior. Furthermore, the changes in these global scores were correlated with
\end{abstract}


changes in the cortisol awakening response $(R=-0.55)$, i.e. the effects of PQQ on improvement of sleep quality are supported by a biomarker.

Keywords: Pyrroloquinoline quinone, stress, fatigue, quality of life, sleep

\section{INTRODUCTION:}

Pyrroloquinoline quinone (PQQ) is an organic molecule discovered in bacteria in 1979 as the third redox coenzyme following nicotinamides (pyridine nucleotides) and flavines PQQ is water-soluble, having 3 carboxyl groups, a quinone, and chemical properties similar to the combined attributes of ascorbic acid, riboflavin, and vitamin $\mathrm{B}_{6}$. PQQ is present in various vegetables and beverages, especially in tea, natto (fermented soybeans), and fruits [1,2], and is also found in the human tissues and fluids, especially breast milk. In animal studies, PQQ as administration influences anti-oxidative capacity, nerve growth factor enhancement and mitochondrial biogenesis [3-7]. Based on such data, we conducted studies that indicate PQQ administration has a positive influence on brain functions, especially short-term memory and attention in elderly healthy volunteers [8]. Accordingly, and to add to our previous observations, we focus herein on mental stress, fatigue, quality of life (QOL) and sleep quality. Adult male and female office workers who complained of fatigue and/or disordered sleep were used as subjects.

\section{SUBJECTS AND METHODS:}

Test substance. The test substance was provided in the form of a hard capsule containing 20 $\mathrm{mg}$ of pyrroloquinoline quinone $\mathrm{Na}_{2}$ salt $\left(\right.$ BioPQQ $^{\mathrm{TM}}$ ) manufactured by Mitsubishi Gas Chemical Co., Inc. (Tokyo, Japan). A capsule was ingested with a cup of water once a day after breakfast. The time of ingestion and the number of capsules ingested were recorded in a diary. The duration of the study was 8 weeks. The dose of $20 \mathrm{mg}$ of PQQ per day was based on our previous clinical study [8].

Study design. The study followed an open-label trial. The effectiveness of PQQ was assessed by comparing the evaluation measures before and after PQQ administration. Primary evaluations consisted of four methods of subjective self-reporting were adopted for evaluating stress, fatigue, QOL and sleep quality. In addition, the increase of cortisol secretion called the cortisol awakening response (CAR) was measured. We adopted CAR as an objective method of evaluation. In general, job stress or general life stress raises the cortisol following 
awakening, while fatigue, burnout or exhaustion reduces that [9]. With respect to sleep, some studies report that high CAR is associated with healthy sleep [10, 11]

Subjects. Seventeen volunteers were selected from adult male and female workers with a diagnosed sleep disorder or complaint and fatigue using as selection criteria the Athens Insomnia Scale (AIS), a scale of insomnia [12], and the Profile of Mood States-Short Form (POMS-S) [13] .

Selection criteria were: (1) men and women aged from 20 to 60; (2) those employed full-time; (3) those who scored over 6 points on the AIS at the examination before enrollment; and (4) those who scored over 50 points in the $\mathrm{T}$ score of fatigue and below 50 points in the $\mathrm{T}$ score of vigor on the POMS-S at the examination before enrollment. Exclusion criteria, discontinuance criteria, dropout criteria, and restrictions were listed in Supporting Information.

The study was conducted in compliance with the "Declaration of Helsinki" and "The Ethical Guideline for Epidemiological Study" (issued in 2004 by the Ministry of Education, Culture, Sports, Science and Technology, and the Ministry of Health, Labour and Welfare). The study (Protocol No.21584) was approved by the clinical study review committee of the Miho Clinic (Tokyo, Japan) on the basis of the protocol and information on the test substance on July 17, 2009. Actual study was done in Ueno Clinic (Tokyo, Japan). The details of this study were explained to the subjects before the start of the study and informed consent given by free will was obtained from each subject.

Study timelines. The subjects visited Ueno Clinic for the first examination. Prior to PQQ intervention, the subjects filled out the POMS-S inventory, the life event survey, the Pittsburgh Sleep Quality Index Japanese version (PSQI-J) and the QOL questionnaire and underwent physical examination (anthropometry, blood pressure and heart rate) and blood and urine tests. Following selections, subjects were next kept overnight in a comfortable setting so that saliva samples could be collected 30 min after awakening to determine the cortisol levels in saliva. The subjects then filled out the Oguri-Shirahata-Azumi Sleep Inventory (Middle Aged and Aged version) (OSA-MA) soon after awakening to evaluate sleep quality during the past one week.

The subjects were then instructed to ingest the test substance and visit the clinic on the weekends of the 1st $(1 w), 2$ nd $(2 w)$, 4th $(4 w)$ and 8th week $(8 w)$ after the initiation of supplementation. On the clinic examination days, they filled out the QOL questionnaire every 4 weeks and the POMS-S every 2 weeks. The POMS-S on the 6 th weekend (6w) was filled out 
at home. An examination of their general condition, including physical examination as well as blood and urine tests, was conducted every 4 weeks. On weekend days, the OSA-MA was filled out soon after awakening at home. Saliva samples were collected soon after and $30 \mathrm{~min}$ after awakening on the latest weekday before the clinic examination day of the weekends of $4 \mathrm{w}$ and $8 \mathrm{w}$. On the examination day, the collected saliva samples were brought to the clinic. The test schedule is shown in Table S1 (see Supporting Information). The duration of this study was from July to November, 2009.

Evaluation of POMS-S and QOL. The effectiveness of PQQ was evaluated using the POMS-S and the QOL Questionnaire to measure the changes at each time point after ingestion and also by the results of intergroup comparison between the values prior to supplementation (the baseline values) and the values at each time point after supplementation.

The POMS Japanese version is a questionnaire comprising 65 questions about six components: tension-anxiety, depression, anger-hostility, vigor, fatigue and confusion [14]. The POMS-S shortens the number of questions from 65 to 30, for ease of use [13]. Despite this, it is highly reliable and produces similar results to the POMS. For each question, the subjects select one of five answers ranging from "not at all" (0 points) to "very frequently" (4 points), according to how many times the mood in question occurred in the past 7 days. All 30 questions are divided into six components with five questions each, and the total points for each component are calculated and designated the raw score. After the raw score is corrected for age and gender, the standardized score ( $\mathrm{T}$ score) is calculated. The higher score for vigor indicates the better condition, while the lower score for the other five components indicates the better condition.

QOL was evaluated by Nagata's QOL Questionnaire [15, 16], scoring measures for appetite, sleep, excretion, urination, exercise, obsession, pain, sexual life, social life, happiness, family life, and fullness and satisfaction in the overall daily life. For each question, the subjects select one of five to twelve answers ranging from "best" (1 points) to "worst" (5 points). In cases of multiple answers, the maximum point score was adapted. For all measures, the lower score indicates the better condition.

Evaluation of sleep. Two subjective methods were employed to enhance the reliability of the results of evaluation: the OSA Sleep Inventory and the PSQI-J, which measured changes at respective examination time points after the start of the ingestion of PQQ, allowing intergroup comparison between the values before ingestion (baseline values) and those after ingestion. 
The OSA-MA consists of 16 questions divided into five factors: Factor I (sleepiness at awakening), Factor II (sleep onset and maintenance), Factor III (nightmare), Factor IV (recovery from fatigue) and Factor V (sleep duration)[17]. The subjects select, soon after awakening in the morning, the best answer to each question from the following: "remarkably", "modestly", "slightly" and "not at all". The answers are converted into Zc points and the average point score for each factor is calculated as the evaluation score of that factor (Factor I: 0-32.3, Factor II: 0-29.6, Factor III: 0-29.5, Factor IV: 0-32.7, Factor V: 0-33.5). For all questions, the higher score means the better condition.

The PSQI is a questionnaire developed in the US for screening of sleep disturbance, composed of 18 questions for the seven components of sleep quality, sleep latency, sleep duration, habitual sleep efficiency, sleep disturbances, use of sleeping medication and daytime dysfunction. In this study, the PSQI-J was used [18-20]. The PSQI global score (from 0 to 21) was obtained by totaling the scores of the seven components (0-3). Higher global scores show more disturbed sleep, with a total score of more than six indicating sleep disturbance. The global score and the scores of the seven components were calculated.

Measurement of salivary cortisol. In the present study, the increase of salivary cortisol level from soon after awakening to $30 \mathrm{~min}$ after awakening was defined as CAR and used to determine the amount of cortisol secretion non-invasively [9].

Samples were collected on the latest weekday before the examination day and on the examination day (weekend) at $0 \mathrm{w}$ (before PQQ ingestion), $4 \mathrm{w}$ and at $8 \mathrm{w}$ by using the saliva sampling tube (Salisoft) (Assist Co., Tokyo, Japan). The times of awakening and sample collection were recorded. Until the completion of sample collection, eating, drinking, smoking and tooth brushing were not permitted. The saliva samples collected were kept in a home refrigerator and brought in a cooler bag with cooling agent to the clinic on examination day. The concentration of salivary cortisol was determined by means of the salivary cortisol EIA kit (Salimetrics) of expanded range and high sensitivity.

Safety evaluation. Adverse events were recorded for subjects who had taken PQQ at least once. Any undesirable subjective or objective symptom was regarded as an adverse event and appropriate treatment was administered if necessary, with recording of details of symptoms and findings, the date of occurrence and the date of resolution. The seriousness of the event, the details of treatment (if treated), the outcome and the cause and effect relationship to PQQ were reviewed and recorded on the case report form. 
The safety of PQQ was evaluated based on the adverse events (subjective symptoms, objective findings and abnormal changes in the measured values) that were judged to be possibly related to $\mathrm{PQQ}$.

Statistical analysis. All measured values and changes were expressed as mean \pm SD. If the analysis of variance by repeated measure revealed a statistically significant difference in the measured value before and after ingestion, the baseline value and the measured values at each examination time after ingestion were compared by the single sample t-test for the POMS-S and OSA-MA. For PSQI-J and QOL, the values were compared by the Wilcoxon signed rank test. The multiple comparisons were made by the Holm's method. For the physical examination values, the changes at each time point after ingestion from the baseline values were evaluated by the single sample t-test. The two-sided level of significance was $5 \%$.

\section{RESULTS:}

\section{Subjects: background information and physical examination.}

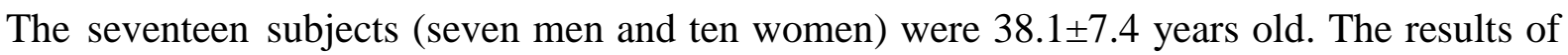
physical examination are presented in Table 1. There was no discontinuance or dropout during the study period. At the time of their selection, the scale for sleeplessness by the AIS was $12.6 \pm 2.8$ and the global score by the PSQI-J was $10.0 \pm 1.9$, demonstrating disordered sleep. The $\mathrm{T}$ score of [Vitality] in the POMS-S was $35.7 \pm 4.6$ and that of [Fatigue] was $71.8 \pm 5.1$, demonstrating stress and fatigue. During the study, none of the subjects had any major changes in employment and working hours or stressful events such as changes in home environment, diseases and birth or death of close relatives.

Table 1. Results of physical examination

\begin{tabular}{lllll}
\hline Measurement & Unit & $0 \mathrm{w}$ & $4 \mathrm{w}$ & $8 \mathrm{w}$ \\
\hline Height & $\mathrm{cm}$ & $163.75 \pm 9.47$ & & \\
\hline Weight & $\mathrm{kg}$ & $58.26 \pm 10.33$ & $58.35 \pm 10.39$ & $58.66 \pm 10.60$ \\
\hline BMI & $\mathrm{kg} / \mathrm{m}^{2}$ & $21.54 \pm 1.93$ & $21.58 \pm 1.93$ & $21.68 \pm 2.03$ \\
\hline Systolic blood pressure & $\mathrm{mmHg}$ & $112.5 \pm 12.2$ & $108.6 \pm 11.4 *$ & $113.0 \pm 11.1$ \\
\hline Diastolic blood pressure & $\mathrm{mmHg}$ & $66.8 \pm 7.2$ & $65.5 \pm 6.1$ & $67.6 \pm 4.3$ \\
\hline Heart Rate & $\mathrm{bpm}$ & $70.1 \pm 7.2$ & $68.1 \pm 10.1$ & $66.9 \pm 6.8$ \\
\hline
\end{tabular}

$n=17$ paired t-test $*: p<0.05$ 


\section{Effects of PQQ on stress, fatigue and QOL.}

The results of POMS-S to evaluate stress and fatigue are presented in Figure 1. The analysis of variance by repeated measures revealed that duration-dependent significant decreases were observed in five components, except vigor, with PQQ ingestion.

The scores of mood states before PQQ ingestion and after eight-weeks of PQQ ingestion are shown in Figure 2. After $8 \mathrm{w}$, the $\mathrm{T}$ score of vigor increased, while those of other five components decreased. These results indicated improvements in stress and fatigue with PQQ.

The results of the evaluation of QOL are shown in Table 2. The four measures of (Appetite, Sleep, Obsession and Pain) significantly declined, indicating significant improvement with PQQ. The three measures of (Fullness of social life, Happiness and fullness in the family life, and Fullness and satisfaction in the overall daily life) showed tended to decline (i.e. improve).
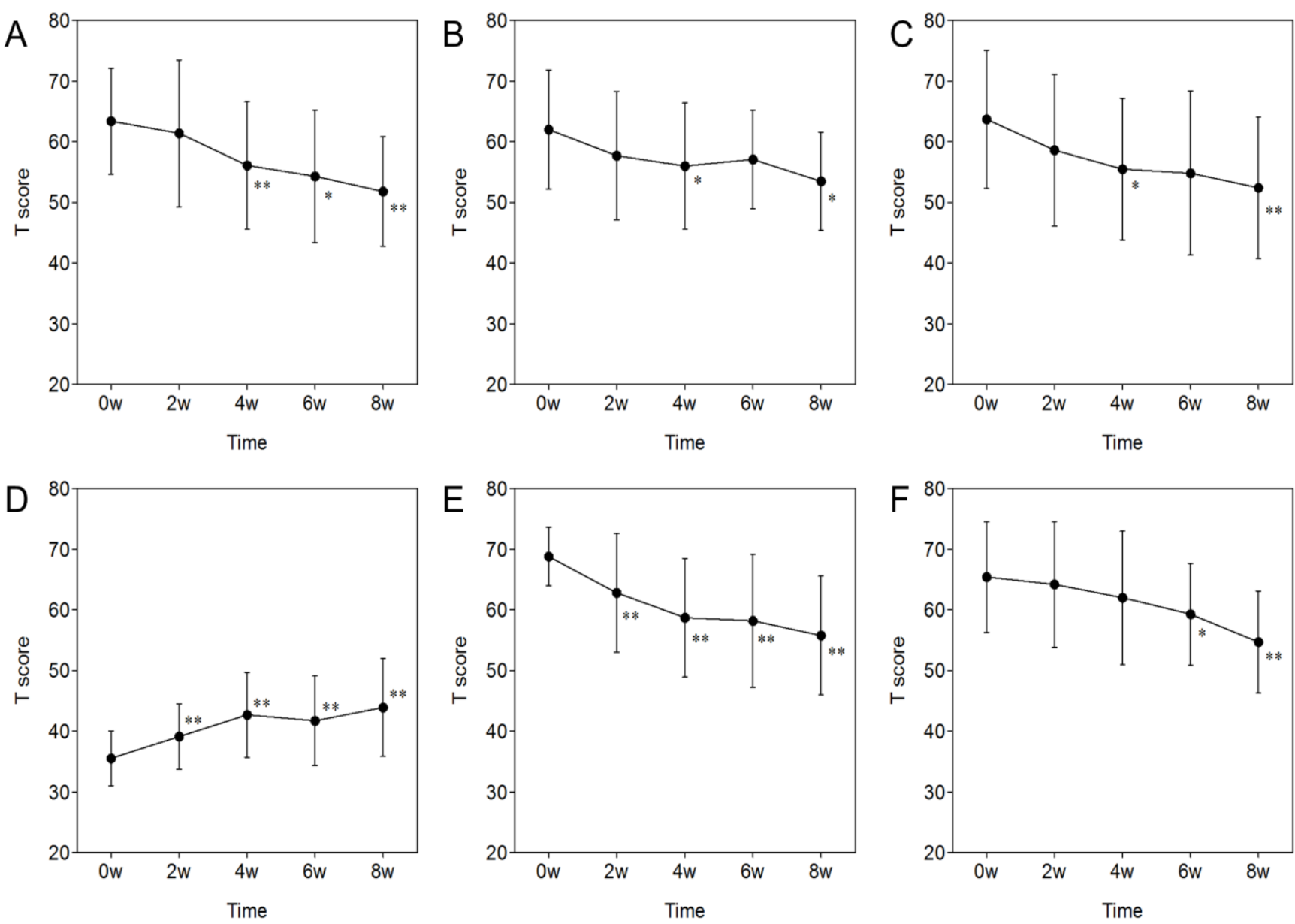

Figure 1. Effects of PQQ supplementation on POMS-S scores of (A) Tension-Anxiety, (B) Depression, (C) Anger-Hostility, (D) Vigor, (E) Fatigue, and (F) Confusion $(n=17)$. Vertical bars indicate standard deviation. (paired t-test $* *: p<0.01, *: p<0.05$ ) 


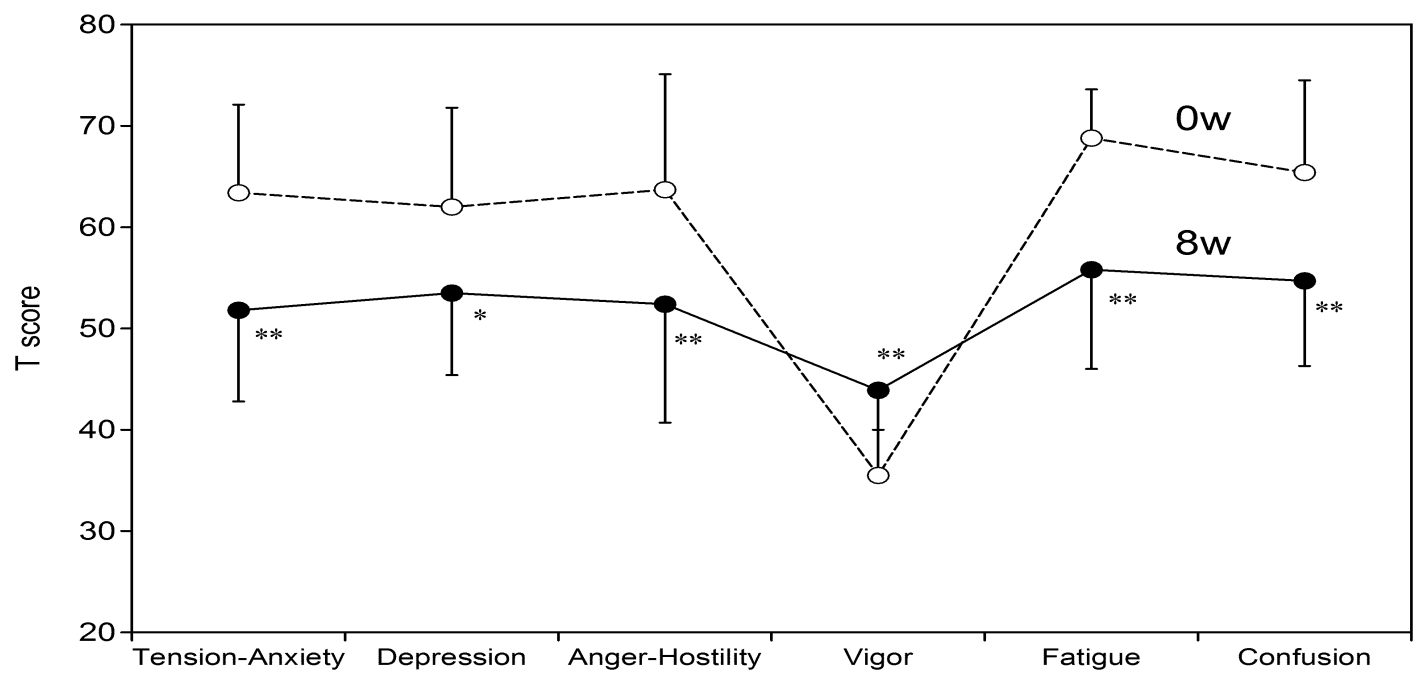

Figure 2. Effects of PQQ supplementation on POMS-S scores $(n=17)$. Vertical bars indicate standard deviation. (paired t-test $* *: p<0.01, *: p<0.05$ ).

Table 2. Effects of PQQ supplementation on QOL scores

\begin{tabular}{clll}
\hline Measurement & $0 \mathrm{w}$ & $4 \mathrm{w}$ & $8 \mathrm{w}$ \\
\hline Appetite & $3.5 \pm 0.9$ & $2.5 \pm 0.9 * *$ & $2.3 \pm 0.7 * *$ \\
\hline Sleep & $4.2 \pm 0.4$ & $3.5 \pm 0.9 *$ & $3.0 \pm 0.9 * *$ \\
\hline Excretion & $3.0 \pm 0.8$ & $2.9 \pm 0.9$ & $2.6 \pm 1.1$ \\
\hline Urination & $3.4 \pm 0.9$ & $2.8 \pm 1.1$ & $2.9 \pm 0.8$ \\
\hline Exercise & $3.4 \pm 1.3$ & $3.6 \pm 1.1$ & $3.6 \pm 1.0$ \\
\hline Obsession & $3.8 \pm 0.8$ & $3.2 \pm 1.3 *$ & $2.5 \pm 1.1 * *$ \\
\hline Pain & $3.6 \pm 0.8$ & $3.3 \pm 0.8 *$ & $2.9 \pm 1.1 *$ \\
\hline Sexual life & $3.8 \pm 1.0$ & $3.6 \pm 1.2$ & $3.6 \pm 1.2$ \\
\hline Fullness of the patient's social life & $3.0 \pm 0.7$ & $2.6 \pm 0.6$ & $2.5 \pm 0.5$ \\
\hline Happiness and fullness in family life & $2.4 \pm 1.1$ & $2.2 \pm 0.9$ & $2.0 \pm 1.0$ \\
\hline Fulfillment and satisfaction in overall & $3.1 \pm 0.4$ & $2.8 \pm 0.6$ & $2.7 \pm 0.6$ \\
daily life & & & \\
\hline
\end{tabular}

$n=17$, Wilcoxon signed rank test (multiple comparison by Holm's test)

$* *: p<0.01, *: p<0.05$

\section{Effects of PQQ on sleep.}

The results of the OSA-MA are shown in Figure 3. PQQ ingestion caused significant duration-dependent improvements in four of the five factors except for Factor III (nightmare). 
Table 3 shows the results of the PSQI-J. The PSQI-J global score went down depending on the duration of PQQ ingestion, as did individual components such as sleep quality, sleep latency, sleep duration and daytime dysfunction.

Finally, to investigate the relation of CAR to sleep, the correlation between CAR and the global score of PSQI-J was assessed. The ten subjects with CAR values below zero at $0 \mathrm{w}$ and 8w were excluded as "non-compliant to protocol", i.e. collection of saliva beyond the 30 minutes post-sleep cut-off $[9,21,22]$ (Table S2, see Supporting Information). In the analyses of seven compliant subjects, the 8-week changes in PSQI scores were correlated with changes in $\operatorname{CAR}(R=-0.55)$ (Figure 4).

Table 3. Effects of PQQ supplementation on sleep (PSQI-J scores)

\begin{tabular}{llll}
\hline Component & $0 \mathrm{w}$ & $4 \mathrm{w}$ & $8 \mathrm{w}$ \\
\hline \hline PSQI-J global score & $10.0 \pm 1.9$ & $8.2 \pm 2.1 * *$ & $6.4 \pm 2.0 * *$ \\
\hline \hline Sleep quality & $2.2 \pm 0.4$ & $1.6 \pm 0.5 * *$ & $1.5 \pm 0.7 * *$ \\
\hline Sleep latency & $2.1 \pm 0.9$ & $1.8 \pm 0.8$ & $1.4 \pm 0.9 * *$ \\
\hline Sleep duration & $2.4 \pm 0.6$ & $2.1 \pm 0.6 *$ & $1.9 \pm 0.6 * *$ \\
\hline Habitual sleep efficiency & $0.5 \pm 0.6$ & $0.2 \pm 0.4$ & $0.1 \pm 0.2$ \\
\hline Sleep disturbance & $1.1 \pm 0.3$ & $1.1 \pm 0.7$ & $0.8 \pm 0.6$ \\
\hline Use of sleeping medication & $0.0 \pm 0.0$ & $0.2 \pm 0.7$ & $0.0 \pm 0.0$ \\
\hline Daytime dysfunction & $1.8 \pm 0.8$ & $1.4 \pm 0.5 *$ & $0.9 \pm 0.7 * *$ \\
\hline
\end{tabular}

$n=17$, Wilcoxon signed rank test (multiple comparison by Holm's test) ${ }^{*}: p<0.01, *: p<0.05$

\section{Safety.}

\section{Subjective symptoms and objective symptoms}

During ingestion of PQQ, six events occurred in five subjects, but were not judged as related to PQQ. These events were the common cold in four subjects including one complaint of abdominal pain and a slight fever in another subject. Symptoms were minor and transient despite continued ingestion of PQQ.

\section{Results of physical examination}

Table 1 shows the changes of mean values and standard deviations obtained from the physical examination. They were judged that all of the changes were clinically negligible. There were no adverse events. Systolic blood pressure was also reduced significantly at $4 \mathrm{w}$, a clinically significant positive effect. 

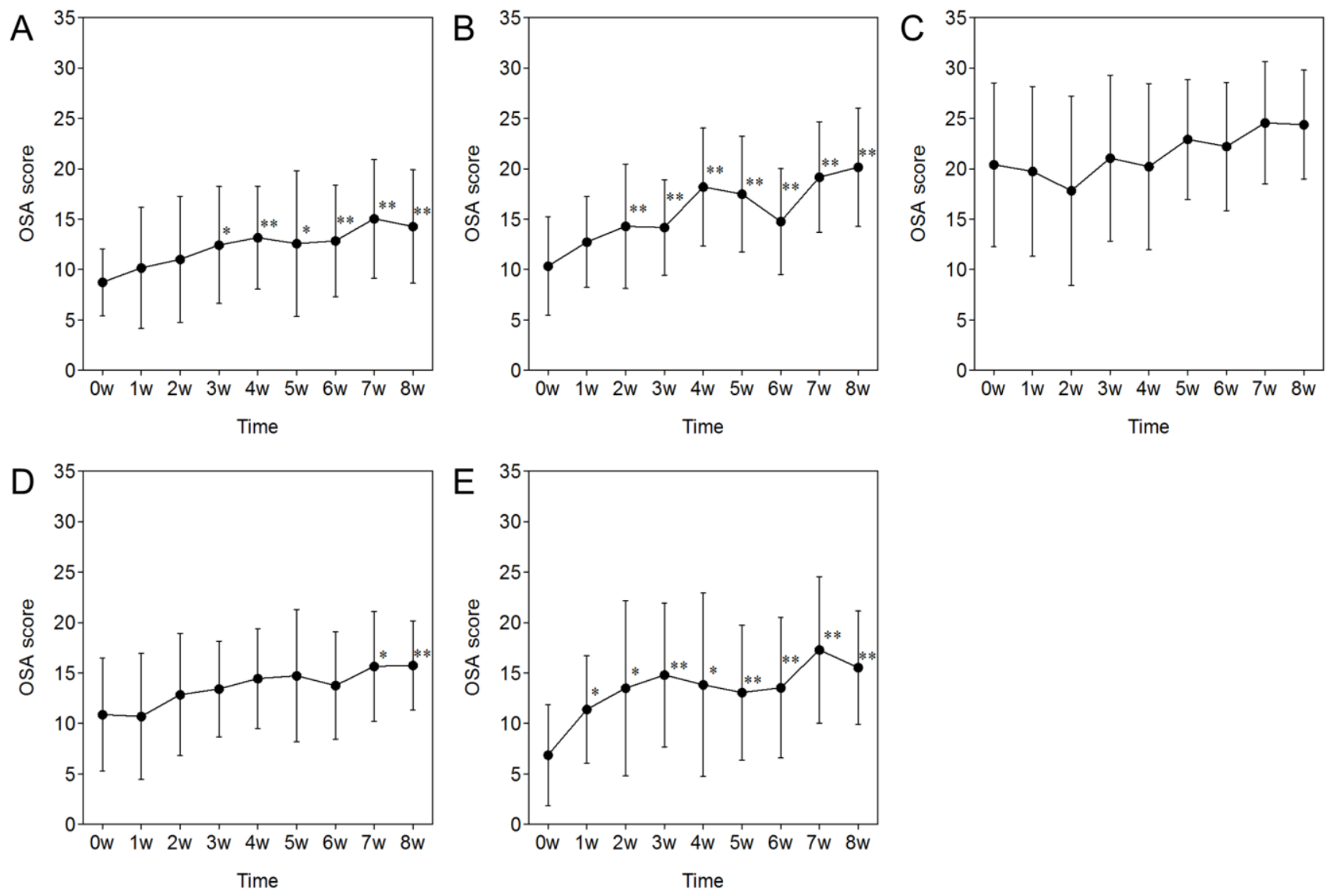

Figure 3. Effects of PQQ supplementation on OSA-MA scores of (A) Factor I (sleepiness at awakening), (B) Factor II (sleep onset and maintenance), (C) Factor III (nightmare), (D) Factor IV (recovery from fatigue), and (E) Factor V (sleep duration) ( $n=$ 17).Vertical bars indicate standard deviation. (paired t-test $* *: p<0.01, *: p<0.05$ ).

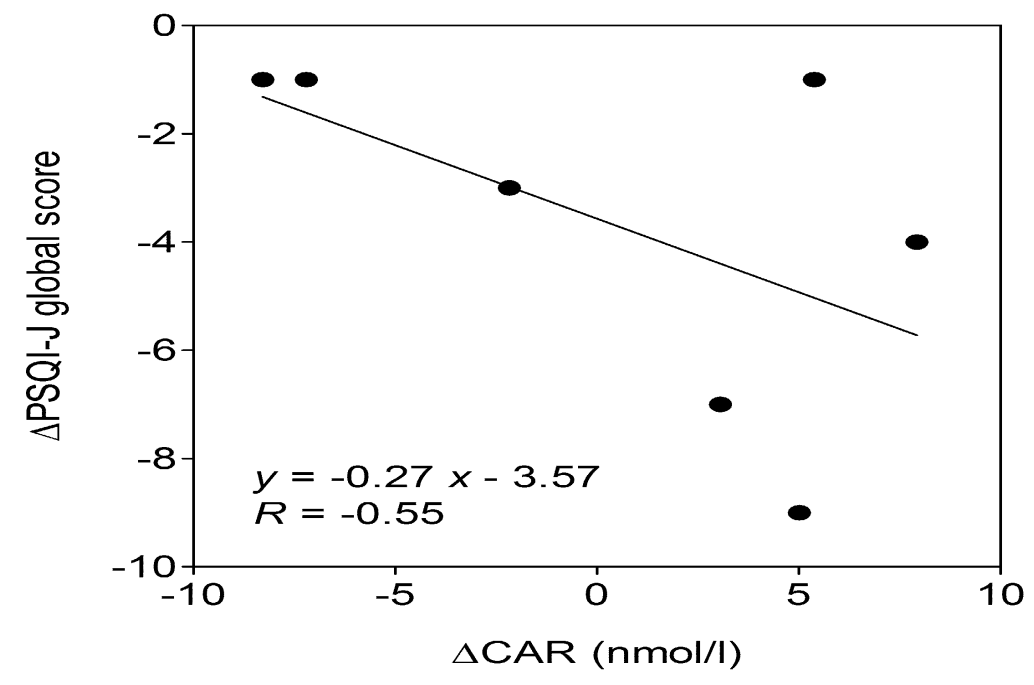

Figure 4. The correlation between changes in weekday CAR and changes in PSQI-J global score after 8 weeks of ingestion $(n=7)$. The line represents a linear regression $(y=-0.27 x$ $-3.57, R=-0.55)$. Raw data represents Table S4. 


\section{DISCUSSION:}

This was the first human study on the effectiveness of oral PQQ supplementation focusing on stress, fatigue, sleep and QOL. Both the OSA-MA and PSQI-J demonstrated significantly improved sleep quality. The concurrent results from two different evaluation methods strongly supported study validity. The results of subjective evaluations by the POMS-S, OSA-MA and PSQI-J suggest that PQQ supplementation also improved sleep, leading to reduced negative mental states, relief of fatigue and rise of positive mood (Table 2).

The scores of the five components, except vigor, in the POMS-S evaluation continued to fall and scores for vigor continued to rise during 8 weeks (Figure 1). Scores of most factors of the OSA-MA showed an upward trend during 8 weeks (Figure 3). These data suggest that PQQ improved not only fatigue but also disordered sleep and QOL.

To examine the physiological basis of this action, we also measured CAR as a biomarker. It has been reported that sleep quality is closely related to CAR [11]. Moreover, in the comparison between primary insomnia patients and healthy volunteers, Backhaus et al. [10] observed that the average cortisol level of the former at awakening was significantly lower than that of the latter. On the other hand, lower PSQI-J global score indicates better sleep quality. In the analysis of our compliant subjects $(n=7)$, a correlation was observed between changes in PSQI-J global score and changes in CAR after 8-weeks of PQQ ingestion (Figure 4 and Table S3, see Supporting Information). These findings suggest that when normal sleep is recovered with PQQ and cortisol secretion due to sleep disturbance is repressed, which occurs with a normal or improved awakening response.

This study is the first human clinical trial on the effect of PQQ on stress, fatigue, and sleep, to better reflect the primary outcome measurements. The results of this trial are therefore, encouraging and should be extended in a placebo-controlled study with a larger study population. The anti-oxidative capacity or mitochondrial biogenesis function of PQQ might be responsible for the outcomes in this study. However, the elucidation of the mode of action is needed in further study.

Conclusions: In conclusion, PQQ supplementation seems associated with improved sleep quality and duration. Mood also was improved by diminishing feelings of fatigue, and improved QOL on measures of appetite, sleep, pain and obsession. The improvement of the PSQI-J global score correlated with changes in CAR. These data indicate that PQQ can be regarded as a potentially useful dietary supplement as it may relate to improving fatigue. Importantly, no health issues were associated with PQQ supplementation. 
Competing interests: The authors have no financial interests or conflicts of interest.

Author's contributions: All authors contributed to this study.

Abbreviations: AIS, Athens Insomnia Scale; CAR, cortisol awakening response; OSA-MA, Oguri-Shirakawa-Azumi Sleep Inventory (Middle Aged and Aged version); POMS-S, Profile of Mood States-Short Form; PQQ, pyrroloquinoline quinone; PSQI-J, Pittsburgh Sleep Quality Index Japanese version; QOL, quality of life;

Acknowledgements: We thank Dr. Robert Rucker for helpful suggestions concerning our manuscript.

\section{REFERENCES:}

1. Rucker R, Stites T, Suzuki Y, Steinberg F, Storms D. Physiological importance of pyrroloquinoline quinone (in Japanese). Vitamin 2001;75(7):381-387.

2. Tsuge H. PQQ (Pyrroloquinoline Quinone) (in Japanese). Vitamin 2005;79(8):383-389.

3. Ohwada K, Takeda H, Yamazaki M, Isogai H, Nakano M, Shimomura M, et al. Pyrroloquinoline Quinone (PQQ) Prevents Cognitive Deficit Caused by Oxidative Stress in Rats. J Clin Biochem Nutr 2008;42:29-34.

4. Ouchi A, Nakano M, Nagaoka S, Mukai K. Kinetic study of the antioxidant activity of pyrroloquinolinequinol (PQQH(2), a reduced form of pyrroloquinolinequinone) in micellar solution. J Agric Food Chem 2009;57(2):450-456.

5. Rucker R, Chowanadisai W, Nakano M. Potential physiological importance of pyrroloquinoline quinone. Altern Med Rev 2009;14(3):268-277.

6. Stites T, Storms D, Bauerly K, Mah J, Harris C, Fascetti A, et al. Pyrroloquinoline quinone modulates mitochondrial quantity and function in mice. $\mathrm{J}$ Nutr 2006;136(2):390-396.

7. Yamaguchi K, Sasano A, Urakami T, Tsuji T, Kondo K. Stimulation of nerve growth factor production by pyrroloquinoline quinone and its derivatives in vitro and in vivo. Biosci Biotechnol Biochem 1993;57(7):1231-1233.

8. Nakano M, Ubukata K, Yamamoto T, Yamaguchi H. Effect of pyrroloquinoline quinone (PQQ) on mental status of middle-aged and elderly persons (in Japanese). Food style 21 2009;13(7):50-53. 
9. Chida Y, Steptoe A. Cortisol awakening response and psychosocial factors: a systematic review and meta-analysis. Biol Psychol 2009;80(3):265-278.

10. Backhaus J, Junghanns K, Hohagen F. Sleep disturbances are correlated with decreased morning awakening salivary cortisol. Psychoneuroendocrinology 2004;29(9):1184-1191.

11. Lasikiewicz N, Hendrickx H, Talbot D, Dye L. Exploration of basal diurnal salivary cortisol profiles in middle-aged adults: associations with sleep quality and metabolic parameters. Psychoneuroendocrinology 2008;33(2):143-151.

12. Soldatos CR, Dikeos DG, Paparrigopoulos TJ. Athens Insomnia Scale: validation of an instrument based on ICD-10 criteria. J Psychosom Res 2000;48(6):555-560.

13. Yokoyama K. POMS Shortened Version-Manual and Commentary on Cases (in Japanese). Tokyo, Kaneko Syoboh 2005:1-9.

14. Yokoyama K, Araki S. POMS Manual (in Japanese). Tokyo, Kaneko Syoboh 1994.

15. Nagata K, Himeno T, Okamoto A, Ito M, Kamano Y, Kaneko M, et al. QOL (Quality of Life), Its Significance in Clinical Evaluation and Practices (in Japanese). J. Clinical Therapeutics \& Medicines 1989;5(2):211-235.

16. Nagata K, Okamoto A, Kamano Y, Kamano S, Yamazaki M, Kubota K, et al. Quality of life variables as new health indicators. In Behavioral medicine: An integrated behavioral approach to health and illness. Elsevier Science Publisher B. V. 1992.

17. Yamamoto Y, Tanaka H, Takase M, Yamazaki K, Azumi K, Shirakawa S. Standardization of revised version of OSA sleep inventory for middle-aged and aged. Brain Science and Mental Health 1999;10(4):401-409.

18. Doi Y, Minowa M, Uchiyama M, Okawa M. Development of the Pittsburgh sleep quality index, Japanese versio (in Japanese). Japanese J. Psychiatric Treatment 1998;13(6):755- 763.

19. Doi Y, Minowa M, Uchiyama M, Okawa M, Kim K, Shibui K, et al. Psychometric assessment of subjective sleep quality using the Japanese version of the Pittsburgh Sleep Quality Index (PSQI-J) in psychiatric disordered and control subjects. Psychiatry Res 2000;97(2-3):165-172.

20. Uchiyama M. Sleep Disturbance: Handling and Guidline of Treatments (in Japanese). Jiho 2002:227-235. 
21. Dockray S, Bhattacharyya MR, Molloy GJ, Steptoe A. The cortisol awakening response in relation to objective and subjective measures of waking in the morning. Psychoneuroendocrinology 2008;33(1):77-82.

22. Kudielka BM, Broderick JE, Kirschbaum C. Compliance with saliva sampling protocols: electronic monitoring reveals invalid cortisol daytime profiles in noncompliant subjects. Psychosom Med 2003;65(2):313-319. 


\section{Supporting Information}

\section{Subjects and Methods}

\section{Exclusion criteria}

1) Those who took routinely the foods or health foods compounded with the active constituent of this test food (PQQ)

2) Those who had regularly any medical treatment (or corresponding treatment) or took medicines or health foods for the purpose of improving sleep, fatigue and stress

3) Those who were engaged to works on a day-night shift or physical labors such as transportation of heavy goods and were working on an irregular schedule

4) Those who did not follow the prescribed procedures for various examinations performed during this study (such as saliva sampling soon after awakening or filling in questionnaires)

5) Those who had diseases under treatment or to be treated, including insomnia

6) Those who had the anamnesis of serious diseases such as diabetes mellitus, hepatic disorders, renal disorders, hypertension, cardiovascular disorders and abnormal glucose tolerance

7) Those who were judged inadequate as the subject from the results of laboratory tests and physical examination performed before ingestion

8) Those who might possibly show allergic reaction to the test food (PQQ or other ingredients)

9) Those who expected to become pregnant during the study and were pregnant or breast-feeding

10) Those who had been already enrolled in another clinical study when they were recruited to this study

11) Those who were judged inadequate as the substance by the principal investigator of this study

\section{Discontinuance criteria}

The principal investigator could discontinue the study for the subject, when the principal investigator judged that the subject in question fell under the following items, and it was treated as a discontinuance case.

1) When the subject might threaten the safety of other subjects

2) When it was difficult to continue this study due to occurrence of any serious clinical abnormality or accident 
3) When any critical or continuous deviation from the protocol was found during the study

4) When the principal investigator decided to discontinue this study

\section{Dropout criteria}

When the subject withdrew from the study due to the personal reason or intention after the subject had agreed to enroll in this study, the principal investigator discontinued soon this study for the subject in question and treated it as a dropout case.

\section{Restrictions}

During the study, every subject had to comply with the following restrictions (including the notices to be followed till the day before examination and those on the day of examination).

1) The lifestyle before the enrollment in this study (eating, drinking, exercise, sleep, smoking, etc.) should be almost kept during the study. Excessive exercise, drinking and eating should be avoided.

2) When any medicine was taken, its name and amount should be recorded on the diary.

3) The test food should be taken every day in the prescribed amount.

4) The diary should be kept every day.

5) The measures to relieve stress should be taken within the ordinary range.

6) On the day before examination, alcohol should not be taken, and hard exercise should be avoided.

7) On the day before visiting the clinical for examination, eating and drinking should be prohibited after 24 o'clock, except drinking water or tepid water.

8) On the day of examination, the subjects should take the prescribed breakfast and thereafter should fast to the end of examination (water or tepid water is accessible).

9) The subjects should fill in the OSA-MA and collect the saliva sample before the time limit soon after awakening on the appointed day for examination. On the day of saliva sampling, eating, drinking, smoking and tooth brushing should not be permitted before the finish of saliva sampling 30 min after awakening. 
Table S1. Test schedule and test item

\begin{tabular}{|c|c|c|c|c|c|c|c|c|c|c|c|}
\hline \multirow{2}{*}{\multicolumn{2}{|c|}{ Test item }} & \multicolumn{2}{|c|}{$\begin{array}{l}\text { Before } \\
\text { ingestion }\end{array}$} & \multicolumn{8}{|c|}{ Duration of ingestion } \\
\hline & & 1 & 2 & $\mathrm{w}$ & w & $\mathrm{W}$ & $\mathrm{W}$ & $\mathrm{W}$ & $\mathrm{w}$ & $\mathrm{w}$ & w \\
\hline \multicolumn{12}{|c|}{ Hospital visiting (holiday) } \\
\hline \multicolumn{12}{|c|}{ Selection of subjects } \\
\hline \multicolumn{12}{|c|}{ Lifestyle questionnaire } \\
\hline \multicolumn{12}{|c|}{ AIS } \\
\hline \multicolumn{12}{|c|}{ Physical examination } \\
\hline \multicolumn{12}{|c|}{ Blood and urine tests } \\
\hline \multirow{3}{*}{ Sleep } & OSA-MA & & & & & & & & & & \\
\hline & PSQI-J & & & & & & & & & & \\
\hline & CAR & & & & & & & & & & \\
\hline \multirow{2}{*}{$\begin{array}{l}\text { Stress } \\
\text { Fatigue }\end{array}$} & POMS & & & & & & & & & & \\
\hline & Life-event survey & & & & & & & & & & \\
\hline \multicolumn{12}{|c|}{ QOL questionnaire } \\
\hline \multicolumn{12}{|c|}{ Ingestion of test substance } \\
\hline \multicolumn{2}{|c|}{ Record in a diary } & & & & & & & & & & \\
\hline
\end{tabular}

$*^{1}$ : Pre-enrollment examination

$*^{2}$ : Pre-ingestion examination 
Table S2. Effects of PQQ supplementation on salivary cortisol at awakening and CAR in compliant seven subjects (per protocol base) (Mean \pm SD)

\begin{tabular}{|c|c|c|c|c|c|c|}
\hline \multicolumn{2}{|c|}{ Measurement } & \multirow{2}{*}{$\begin{array}{l}\text { Unit } \\
\mathrm{nmol} / \mathrm{l}\end{array}$} & \multirow{2}{*}{$\begin{array}{l}0 w \\
8.9 \pm 4.3\end{array}$} & \multirow{2}{*}{$\frac{4 w}{11.4 \pm 5.4}$} & \multicolumn{2}{|l|}{$8 w$} \\
\hline \multirow{3}{*}{ Weekday } & at awakening & & & & $10.4 \pm 5.8$ & \\
\hline & 30 min after awakening & nmol/l & $14.7 \pm 7.2 \#$ & $13.7 \pm 7.0$ & $16.7 \pm 8.3 \#$ & 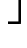 \\
\hline & CAR & $\mathrm{nmol} / \mathrm{l}$ & $5.8 \pm 4.7$ & $2.3 \pm 8.7$ & $6.3 \pm 4.8$ & \\
\hline \multirow{3}{*}{ Weekend } & at awakening & nmol/1 & $11.5 \pm 4.7$ & $9.9 \pm 5.0$ & $10.6 \pm 6.6$ & \\
\hline & 30 min after awakening & $\mathrm{nmol} / \mathrm{l}$ & $17.4 \pm 9.1 \#$ & $11.9 \pm 7.9$ & $16.9 \pm 8.9 \#$ & \\
\hline & CAR & $\mathrm{nmol} / \mathrm{l}$ & $5.9 \pm 5.0$ & $1.9 \pm 5.8$ & $6.3 \pm 4.6$ & \\
\hline
\end{tabular}

Significant difference between salivary cortisol at awakening and salivary cortisol $30 \mathrm{~min}$ after awakening (paired t-test) $\quad \#: p<0.05$.

Table S3. Raw data of Figure 4

\begin{tabular}{ccccccc}
\hline & \multicolumn{3}{c}{ Weekday CAR (nmol/l) } & \multicolumn{5}{c}{ PSQI-J global score } \\
Subject No. & $0 \mathrm{w}$ & $8 \mathrm{w}$ & $\Delta \mathrm{CAR}$ & & \multicolumn{1}{c}{$\Delta$ PSQI-J global score } \\
& & & & $0 \mathrm{w}$ & $8 \mathrm{w}$ & \\
\hline 1 & 0.2 & 8.1 & 7.9 & 7 & 3 & -4 \\
2 & 13.1 & 4.8 & -8.3 & 9 & 8 & -1 \\
3 & 5.0 & 8.1 & 3.1 & 11 & 4 & -7 \\
4 & 8.2 & 1.0 & -7.2 & 10 & 9 & -1 \\
5 & 9.6 & 14.6 & 5.0 & 13 & 4 & -9 \\
6 & 1.5 & 6.9 & 5.4 & 9 & 8 & -1 \\
7 & 2.8 & 0.7 & -2.1 & 10 & 7 & -3 \\
\hline
\end{tabular}

